\title{
Nursing workforce standards and planning in Australian intensive care units
}

Ged Williams RN, RM, Crit. Care Cert., Grad. Cert. PSM, Bach. App. Sc. (Adv. Nursing), Grad. Cert Law, LLM (QUT), MHA (UNSW), FRCNA, FCN NSW, FACHSE Divisional Director of Nursing, Royal Melbourne Hospital, Melbourne Health and Adjunct Associate Professor of Nursing, University of Melbourne, Victoria, Australia.

E-mail: Ged.Williams@mh.org.au

Key words: Australia intensive care management planning staffing workforce

\section{SUMMARY \\ * Identifying the right number and skill mix of intensive care nurses has been an inexact science for some time and will probably remain so. \\ * This paper describes guidelines developed in Australia to help illustrate the nursing staff requirements for intensive care in that country. \\ * The methodology was developed using existing documented standards and clarification provided by an expert panel of 16 respected critical care nursing leaders. \\ * It is acknowledged that staffing methods have cultural and context variability, making the application of universal guidelines difficult if not impossible. However, the methodology reported in this paper has the flexibility to be manipulated for various settings and contexts.}

\section{INTRODUCTION}

This paper describes the methodology and findings of the seminal paper developed by Williams and Clarke (2001) to inform current and projected nursing workforce needs for intensive care units in Australia. The method and findings have been used by the Australian College of Critical Care Nurses (ACCCN, 2002) to establish a position paper on intensive care nursing staffing and by the Australian Health Workforce Advisory Committee (AHWAC - Federal Government, 2002) to plan strategically for the needs of the critical care nursing workforce in the first decade of the $21 \mathrm{st}$ century.

\section{BACKGROUND}

In 2001, Williams and Clarke provided a specific methodology to determine the number of nurses required to staff Australia's intensive care unit (ICU) beds. The key questions considered by the approach include:

* What is the average number of full-time equivalent (FTE) nurses required to staff an intensive care unit bed?

* How many full-time equivalent nurses are required to staff intensive care unit beds in Australia?

* How many post-graduate intensive care unit course qualified and unqualified nurses (FTE)

are required to staff Australia's intensive care unit beds? * What is the required replacement factor of intensive care unit nurses per year (qualified and unqualified) to manage the attrition rate?

The approach used minimum standards determined by the Faculty of Intensive Care, the Australian and New Zealand College of Anaesthetists (FICANZCA, 1997), the Australian Council of Healthcare Standards (ACHS, 1997), and consensus views from a panel of 16 critical care nursing experts from around Australia.

\section{METHODOLOGY}

The methodology was supported using empirical data reported by Anderson and Hart (2000) for Australian \& New Zealand Intensive Care Society, and the Australian Institute of Health and Welfare (2000) nurse labour force surveys. Where no empirical data was available, consensus was sought from a panel of 16 critical care nursing leaders. There were two leaders from each State and Territory. They included clinical, management and educational leaders from rural, metropolitan, paediatric, general and cardiac units. Consensus was gained in regard to staffing ratios (registered nurses to intensive care unit and high dependency unit beds), use of supernumerary (ACCESS) nurses, attrition rates, minimum and optimum qualified staff ratios, the number of nurse managers and number of nurse educators required.

\section{Variables identified}

In determining an average full-time equivalent nurse ratio per bed, a number of variables were identified. These included the use of a ratio for registered nurse to intensive care unit patient and high dependency patient (based on the minimum standards of both the Faculty of Intensive Care, Australian and New Zealand College of Anaesthetists, and the Australian Council of Healthcare Standards). Other variables used to determine average full-time equivalent requirements per bed included number of nurse hours per week, number of supernumerary (ACCESS) nurse hours per week and industrial award leave cover. The number of required hours per intensive care unit bed per week was then derived. This was divided by 38 hours to provide a full-time equivalent requirement -38 hours being the number of hours worked by a full time 


\begin{abstract}
ANZICS Database 6
ICUs (adult and paediatric, public and private)

Total physical bed capacity*

Total available ICU bedst

ICU available (open) beds for ventilation $\Delta$

Non-ventilated (assumed high dependency patient) beds

\section{AlHW Study 7}

Average hours per week worked (ICU nurse)

Nurses working in ICU capability that is fully staffed and funded.

$\Delta$ Ventilated bed: a physical intensive care unit bed plus ventilator.

Table 1: Demographics of intensive care units in Australia 1997/8

nurse in Australia.

All variables described were adjustable, particularly the ratio of ACCESS nurses per bed and leave cover ratio. The nurse hours per week were also adjustable according to the average number of hours that units operate. In the case of public hospital intensive care units, they are operational 24 hours a day, 7 days a week. Private hospitals may differ in their operational hours per week and occupancy rates. These factors can be incorporated to adjust the full-time equivalent requirement.

As there was no information to accurately describe the availability of high dependency beds for use in intensive care, the proportion of non-ventilated beds were used as a proxy measurement (see Table 1).
\end{abstract}

\section{Number}

153

1,646

1,420

1,010

410

\section{Number}

33.3

9,922

* Physical beds: a single patient care location fully configured to intensive care unit standards; it is an actual bed, not a bed space.

+ Available (open) beds: beds in use or immediately available for use by admitted patients as required. In intensive care unit this refers to a bed with advanced life support

\section{Using the Williams and Clarke method}

The Williams and Clarke (2001) method also included an estimation of the number of management and clinical educator registered nurses for intensive care. The number of nurse managers was estimated by applying one for each intensive care unit, or by applying an agreed ratio e.g. one for every 12 beds. The number of clinical educator registered nurses was estimated by applying a ratio of one full-time equivalent educator to every 50 registered nurses (headcount) or another agreed ratio e.g. one full-time equivalent for every intensive care unit. A total number of registered nurses required was determined by adding the total number of bedside registered nurses to the total number of management and clinical educators.

The total required headcount forms the basis for an assessment of the current supply by comparing it to the actual headcount (as reported by Australian Institute of Health and Welfare (2000) nurse labour force survey data). Estimating the required number of new entrants per year was possible by applying an attrition rate to the total headcount. The method also allowed the determination of numbers of qualified staff (by applying the desired ratio of post graduate qualified nurses to the overall required headcount) and the number of new qualified staff required each year by applying the desired ratio to the attrition rate.

Using the Williams and Clarke methodology, a full-time equivalent of 6.7 nurses for intensive care unit beds and 3.89 for high dependency beds was calculated (assuming at least 50\% critical care nurses are qualified). This full-time equivalent was then applied to intensive care unit bed numbers accordingly and a total full-time equivalent requirement was determined. Using average hours worked per week, the full-time equivalent requirement was converted to a headcount and provided a baseline critical care nurse requirement for bedside critical care nurses across Australian intensive care units. An additional headcount requirement was determined for critical care nurse educators and managers (see Figures 1-3).

To determine the number of registered nurses needed to staff intensive care unit beds and high dependency unit beds, Williams and Clarke (2001) made a series of assumptions:

* An intensive care unit bed (ventilated) requires a nurse to patient ratio of $1: 1(2,4,5)$;

* A high dependency bed (in an intensive care unit) requires a nurse to patient ratio of at least $1: 2$ $(2,4,5)$. Because of the potential for instability, the ratio of ACCESS* nurse to patient is the same for high dependency patients as for ventilated

patients;

* The ratio of ACCESS* nurse to patient for national measurement is 1:5 (consensus);

* A full nursing day adds up to 26 hours (to allow for handover period/shift changes);

* Industrial award leave entitlements require staff replacement of 1:6 - one nurse on leave for every six currently working (for every six full-time equivalent positions, one extra full-time equivalent is required to cover leave entitlements);

* A full-time equivalent registered nurse works 38 hours per week.

Using these assumptions a series of clacualtions was made (see Figures 1-3)

\section{DISCUSSION}

The method and findings established by Williams and Clarke (2001) were popular with nurses in many intensive care units around Australia. Reasons for this may be that the method was simple to use and apply, appeared to reflect common practice in many units already and was supported by a panel of respected critical care nursing leaders from across the country.

The method and findings used by Williams and Clarke were quickly adopted into a position statement by the Australian College of Critical Care Nurses, the peak nursing body representing critical care nurses in Australia. The position statement on intensive care nursing staffing is replicated in Appendix 1.

The Australian Health Workforce Advisory Committee published 


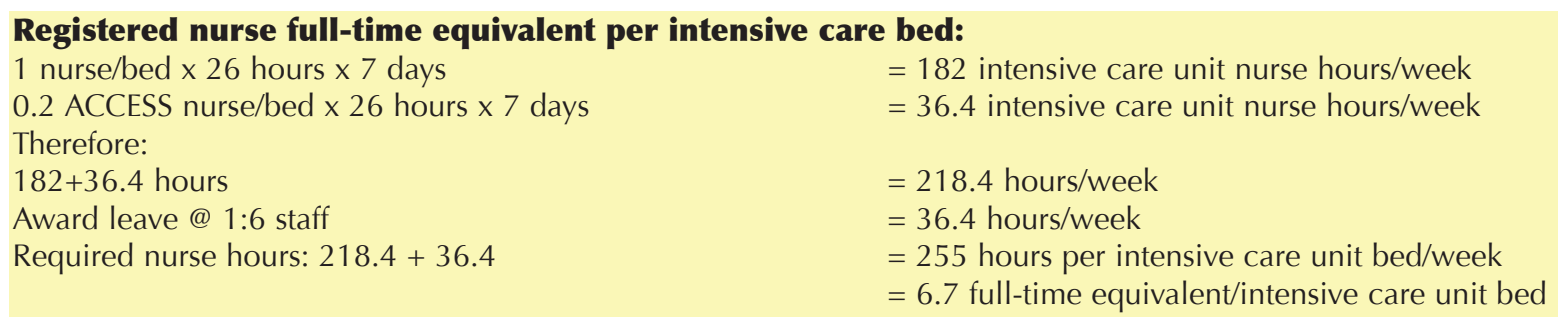

\section{Registered nurse full-time equivalent/step down (high dependency unit) bed:}

0.5 nurse/bed $\times 26$ hours $\times 7$ days

0.2 Access nurse/bed $\times 26$ hours $\times 7$ days

Therefore:

$91+36.4$ hours

Award leave @ 1:6 staff

\section{Required nurse hours:}

$127+21$

= 91 intensive care unit nurse hours/week

$=36.4$ intensive care unit nurse hours/week

$=127$ hours/week

$=21$ hours/week

$=148$ hours per high dependency unit bed/week

$=3.89$ full-time equivalent/high dependency unit bed

*The ACCESS nurses provide 'on-the-floor' Assistance, Co-ordination, Contingency (for a late admission on the shift or staff sick mid-shift), Education (of junior staff, relatives and others), Supervision and Support.

Figure 1: Application of the Williams and Clarke (2001) method to the Australian intensive care nursing workforce.

255 hours per intensive care patient bed/week x 1,010 beds

$148 \mathrm{hrs}$ per high dependency patient bed/week $\times 410$ beds

\section{Subtotal:}

Average nurse works $33.3 \mathrm{hrs} /$ week - total nurses (not FTE)

Educators required@1:50 nurses

NUMs required (153 units)

Total ICU nurses required for available ICU beds:
$=257,550$ hours

$=60,680$ hours

318230 hrs (8347 FTE)

$=9556$ nurses

$=191$ educators

= 153 NUM

9900 RNs

Figure 2. Full-time equivalent nurses required to staff available intensive care unit beds in Australia

255 hrs per intensive care patient bed $\times 1171$

148 hrs per high dependency patient bed x 475

\section{Subtotal:}

Average nurse works $33.3 \mathrm{hrs} /$ week - total nurses (not FTE)

Educators required@ 1:50 nurses

NUMs required (153 units)

Total ICU nurses required for physical ICU beds:
$=298,605 \mathrm{hrs}$

$=70,300 \mathrm{hrs}$

$\mathbf{3 6 8 , 9 0 5}$ hrs (9708 FTE)

$=11,078$ nurses

$=222$ educators

$=153 \mathrm{NUMs}$

11453 RNs

Figure 3. Full-time equivalent nurses required to staff all physical intensive care unit beds in Australia.

a comprehensive study into critical care nursing in Australia 20012011, in which they used the Williams and Clarke methodology to calculate projected requirements for the future critical care nursing workforce across Australia.

The greatest value of this method is that the assumptions can be changed to suit critical care nursing workforces in other countries, or it can be used for nursing workforce projections for groups other than critical care nurses.

As concluded in the Williams and Clarke paper, 'further development and research into "global" staffing methodologies similar to those used in this study to improve the specificity and accuracy of our work and to expand such methods to other fields of nursing' are required...this is but the beginning of the journey.

\section{REFERENCES}

Anderson, T \& Hart, G. (2000) ANZICS Intensive Care Survey 1998: An Overview of Australian and New Zealand Critical Care Resources. ANZICS.

Australian College of Critical Care Nurses. (2002) Position Statement on Intensive Care Nursing Staffing. Australian Critical Care 15 (1): 6-7.

Australian Council on Healthcare Standards. (1997) Guidelines for Intensive Care Units. ACHS, Sydney.

Australian Health Workforce Advisory Committee. (2002) The Critical Care Nurse Workforce in Australia 2001-2011. AHWAC Report, Sydney, Australia.

Australian Institute of Health and Welfare. (2000) Nursing Labour Force 1999. AIHW Cat No. HWL 18, Canberra, Australia.

Faculty of Intensive Care, Australian and New Zealand College of 
Anaesthetists. (1997) Minimum Standards for Intensive Care Units.

Policy document IC-1. FICANZCA, Melbourne, Australia.

Williams, GF \& Clarke, T. (2001) A consensus driven method to measure

the required number of intensive care nurses in Australia. Australian

Critical Care 14 (3): 106-115.

Appendix 1. Position statement on intensive care nursing staffing.

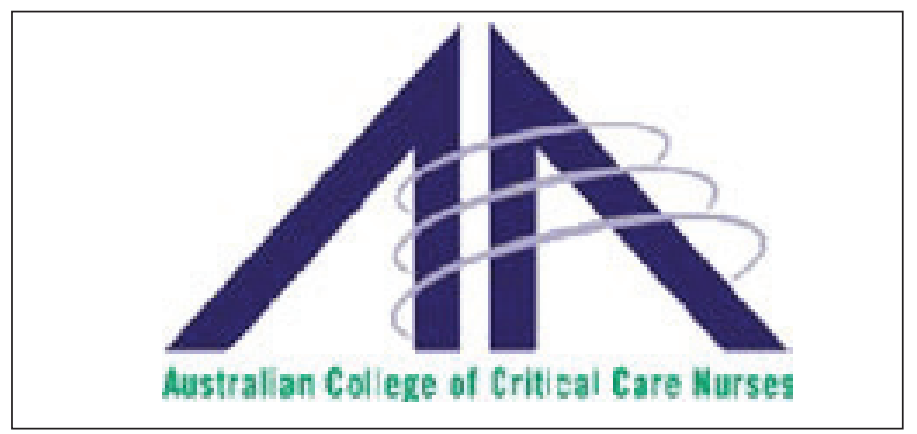

The Australian College of Critical Care Nurses Ltd. (ACCCN) is the peak professional nursing association representing critical care nurses throughout Australia. This position statement outlines the appropriate nursing staffing standards in Australia for Intensive Care Units, taking into account accepted minimum national standards, best practice evidence and a rational economic health and government environment.

ACCCN recommends the following 10 key points and principles to meet the expected standards of critical care nursing in Australia. These standards articulate with those guidelines outlined by both the Australian Council of Healthcare Standards (ACHS, 1997) and the Australian and New Zealand College of Anaesthetists (ANZCA, 1997).

1. Intensive care unit patients (clinically determined) - require a standard nurse/patient ratio of at least 1:1.

2. High dependency patients (clinically determined) - require a standard nurse/patient ratio of at least 1:2

3. Clinical Co-ordinator (team leader) - there must be a designated critical care qualified senior nurse per shift who is supernumerary and whose primary role is responsibility for the logistical management of patients, staff, service provision and resource utilisation during a shift. This includes co-ordinating staff, ensuring compliance with hospital policy and procedures, liaison with medical and allied staff to formulate patient clinical management plans, monitor appropriateness and effectiveness of clinical care, and ensure a safe conducive environment is maintained. This nurse should be guaranteed to be supernumerary for the entire shift.

4. ACCESS nurses: These nurses are in addition to bedside nurses, clinical co-ordinator, unit manager, educators and non-nursing support staff. The ACCESS nurses provide 'on-the-floor' Assistance, Co-ordination, Contingency (for a late admission on the shift or staff sick mid-shift), Education (of junior staff, relatives and others), Supervision and Support. The ACCESS nurse would reduce entry block to intensive care unit for emergency admissions.

ACCCN acknowledges that similar positions have varying names and descriptions in units all over Australia (e.g. float nurses, 'bay nurse', admission nurses).

The role of the ACCESS nurse may be incorporated into the Clinical Co-ordinator's role. However, the Clinical Co-ordinator should not be the only contingency nurse available for emergency admissions. That is, where a unit has the number of beds/qualified staff to justify only one ACCESS nurse, a supernumerary Clinical Co-ordinator must also be rostered on duty.

The ratio of ACCESS nurses required per unit/per shift will depend on the average level of skill and expertise of the total team. As a fair measure of an individual unit's need for ACCESS nurses, ACCCN have linked the required ratio of ACCESS nurses to the overall percentage of qualified critical care nurses available on the roster. Therefore:

Units with $<50 \%$ qual. ICU nurses - 1:4. i.e. one ACCESS nurse for every 4 patients/shift.

Units with 50-75\% qual. ICU nurses - 1:6. i.e. one ACCESS nurse for every 6 patients/shift.

Units with $>75 \%$ qual. ICU nurses -1 :8. i.e. one ACCESS nurse for every 8 patients/shift.

ACCCN acknowledges the crucial support agency/casual nursing staff provide. However, agency/casual staff require additional orientation, support and guidance further emphasising the need for ACCESS nurse positions. ACCCN acknowledges that a combination of both suitable critical care experience and a post-graduate specialist qualification, provide the optimal critical care nursing preparation.

Idiosyncrasies and special needs: In units which have idiosyncratic needs such as retrieval services, large teaching courses, dedicated equipment nurses and major research projects, additional nursing requirements will need to be factored in to the total establishment in addition to that which is described above.

5. At least one designated Nursing Manager (NUM/CNC/NPC/ $\mathrm{CNM}$ or equivalent title) is required per intensive care unit, who is formally recognised as the unit nurse leader. In certain circumstances,

(e.g. large units of $20+$ beds) alternative supports will be required, and these need to be planned independently and in addition to the ratios described above.

6. At least one designated Clinical Nurse Educator (CNE) should be available in each unit. The recommended ratio is one full-time equivalent CNE for every 50 nurses on the intensive care unit roster, with additional educators to run and manage tertiary-based critical care nursing courses. The intensive care unit Clinical Nurse Educator is for unit-based education and staff development activities only and must be located in the intensive care unit itself.

The role of Clinical Nurse Consultant differs between states ranging from unit management to providing a global critical care resource, education and leadership to specific units, hospital and area wide services and to the tertiary education sector.

7. ACHS guidelines (1997) state that intensive care units must have a minimum $\mathbf{5 0} \%$ qualified critical care nurses. ACCCN supports this as a minimum standard, however we assume that the optimum qualified critical care nurse ratio should be $75 \%$. (Units with less than $50 \%$ qualified staff will need additional ACCESS nurses as described in 4 above.) To ensure at least $50 \%$ of intensive care unit nursing staff are qualified (optimally $75 \%$ ), ACCCN recommend that nursing staff without post graduate qualifications should receive financial assistance and study leave to complete a recognised critical care nursing 
course and that such support is factored into the unit budget each year.

8. Resources are allocated to support nursing time and costs associated with quality assurance activities, nursing and multidisciplinary research and conference attendance.

9. Intensive care units are provided with adequate administrative staff, ward assistants, manual handling assistance/equipment, cleaning and other support staff to ensure that such tasks are not the responsibility of nursing personnel. ACCCN believes that the value and cost of intensive care unit nurses does not support their time being used for clerical and cleaning purposes except on very few occasions when the nature of such work is specialised and requires educated or professional knowledge and skill.

10. Senior nursing staff (e.g. CNS) should work towards becoming an Australian Credentialed Critical Care Nurse for which they must be remunerated to a significantly higher level than that of the base grade award.

\section{References:}

Australian Council on Healthcare Standards (1997). Guidelines for Intensive Care Units. Sydney: Australian Council on Healthcare Standards.

Faculty of Intensive Care, Australian and New Zealand College of Anaesthetists (1997). Minimum Standards for Intensive Care Units. IC-1. Melbourne. 